\title{
GENERALIZED $r$-CONVEX FUNCTIONS AND INTEGRAL INEQUALITIES
}

\section{MUHAMMAD ASLAM NOOR*, KHALIDA INAYAT NOOR, FARHAT SAFDAR}

\author{
Department of Mathematics, COMSATS University Islamabad, Islamabad, Pakistan
}

${ }^{*}$ Corresponding author: noormaslam@gmail.com

\begin{abstract}
In this paper, we introduce and investigate a new class of generalized convex functions, known as generalized $r$-convex function. Some new Hermite-Hadamard integral inequalities via generalized $r$-convex functions have been established. Results proved in this paper can be viewed as significant new contributions in this area of research.
\end{abstract}

\section{INTRODUCTION}

In order to obtain some desirable results in general framework related to pure and applied sciences, the concept of convexity has been extended and generalized in several directions, see [1,2,4,5,7,11-17]. Several branches of mathematical and engineering sciences has been developed by using the crucial and significant concepts of convex analysis and hence it becomes one of the most interesting and useful concept of mathematics for last few decades.

Hermite-Hadamard inequality is one of the most important inequality related to convex function, see $[9,10]$. In recent years, much attention has been given to derive the Hermite-Hadamard type inequalities for various types of convex functions, see [8-10,17, 18,22]. Gill et al [8] introduced and investigated the concept of $r$-convex functions. They established some new Hermite-Hadamard integral inequalities for $r$ convex functions. For the applications of $r$-convex functions in statistics and probability theory, see Pecaric et al. $[25-27,30,31]$ and the references therein.

Received 2018-02-07; accepted 2018-04-27; published 2018-09-05.

2010 Mathematics Subject Classification. 26D15, 26D10, 90C23.

Key words and phrases. Generalized convex functions; generalized $r$-convex functions; Hermite-Hadamard type inequalities. (c)2018 Authors retain the copyrights of their papers, and all open access articles are distributed under the terms of the Creative Commons Attribution License. 
Gordji et al. [7] has introduced an other class of convex functions, which is called generalized convex( $\varphi$-convex ) functions. The generalized convex functions are nonconvex functions. For recent developments, see $[3,6,18-21,25-31]$ and the references therein. Inspired and motivated by the ongoing research, we introduce a new class of generalized convex functions, which is called generalized $r$-convex functions. We derive some new Hermite-Hadamard integral inequalities for these nonconvex functions. Some special cases are also discussed, which can be obtained from our main results. The ideas and techniques of this may be starting point for further research in this field.

\section{Preliminaries}

Let $I=[a, b]$ be an interval in real line $\mathbb{R}$. Let $f: I \rightarrow \mathbb{R}$ be a continuous function and $\eta(\cdot, \cdot): \mathbb{R} \times \mathbb{R} \rightarrow \mathbb{R}$ be a continuous bifunction. We need the following well known results and concepts.

Definition 2.1. [7] A function $f: I=[a, b] \rightarrow \mathbb{R}$ is said to be generalized convex function with respect to a bifunction $\eta: \mathbb{R} \times \mathbb{R} \rightarrow \mathbb{R}$, if

$$
f((1-t) a+t b) \leq(1-t) f(a)+t[f(a)+\eta(f(b), f(a))], \forall a, b \in I, t \in[0,1] .
$$

Definition 2.2. A function $f: I=[a, b] \rightarrow \mathbb{R}$ is said to be generalized $r$-convex function with respect to a bifunction $\eta: \mathbb{R} \times \mathbb{R} \rightarrow \mathbb{R}$, if $\forall a, b \in I, t \in[0,1]$

$$
f((1-t) a+t b) \leq \begin{cases}\left\{(1-t)[f(a)]^{r}+t[f(a)+\eta(f(b), f(a))]^{r}\right\}^{\frac{1}{r}}, & r \neq 0, \\ {[f(a)]^{1-t}[f(a)+\eta(f(b), f(a))]^{t},} & r=0 .\end{cases}
$$

1-convex is called generalized convex functions and 0-convex is called generalized log convex functions.

If $t=\frac{1}{2}$, then

$$
f\left(\frac{a+b}{2}\right) \leq \begin{cases}\left\{\frac{[f(a)]^{r}+[f(a)+\eta(f(b), f(a))]^{r}}{2}\right\}^{\frac{1}{r}}, & r \neq 0, \\ \sqrt{[f(a)][f(a)+\eta(f(b), f(a))]}, & r=0 .\end{cases}
$$

The function $f$ is called generalized Jensen $r$-convex function.

If $\eta(f(b)-f(a))=f(b)-f(a)$, then

Definition 2.3. [8] A function $f: I=[a, b] \rightarrow \mathbb{R}$ is said to be $r$-convex function, if

$$
f((1-t) a+t b) \leq \begin{cases}\left\{(1-t)\left[f^{r}(a)\right]+t\left[f^{r}(b)\right]\right\}^{\frac{1}{r}}, & r \neq 0 \\ {[f(a)]^{1-t}[f(b)]^{t},} & r=0 .\end{cases}
$$


Note that for $r=1$, we have classical convex functions and for $r=0$, we have $\log$ convex functions. For the recent applications of convex functions and their generalizations, see [1-9, 12-15, 17, 18, 22-27,31].

The generalized logarithmic means of order $r$ of positive numbers $a, b$ is defined by

$$
L_{r}(a, b)= \begin{cases}\frac{r}{r+1} \frac{a^{r+1}-b^{r+1}}{a^{r}-b^{r}}, & r \neq 0,-1, a \neq b, \\ \frac{a-b}{\log a-\log b}, & r=0, a \neq b, \\ a b \frac{\log a-\log b}{a-b}, & r=-1, a \neq b, \\ a, & a=b .\end{cases}
$$

Definition 2.4. The beta function, also called the Euler integral of the first kind, is defined as

$$
\beta(x, y)=\int_{0}^{1} t^{x-1}(1-t)^{y-1} \mathrm{~d} t=\frac{\Gamma(x) \Gamma(y)}{\Gamma(x+y)}, \quad x, y>0
$$

where $\Gamma($.$) is a Gamma function.$

\section{MAin RESULTS}

In this section, we establish several new integral inequalities of Hermite-Hadamard type for generalized $r$-convex functions.

Theorem 3.1. Let $f: I \rightarrow \mathbb{R}$ be generalized $r$-convex function on $I$. Then for $0<r \leq 1$, we have

$$
\frac{1}{b-a} \int_{a}^{b} f(x) \mathrm{d} x \leq\left(\frac{r}{r+1}\right)\left\{\left([f(a)]^{r}+[f(a)+\eta(f(b), f(a))]^{r}\right)\right\}^{\frac{1}{r}} .
$$

Proof. Let $f$ be generalized $r$-convex function on $I$. Then, $\forall a, b \in I, t \in[0,1]$, we have

$$
f((1-t) a+t b) \leq\left\{(1-t)[f(a)]^{r}+t[f(a)+\eta(f(b), f(a))]^{r}\right\}^{\frac{1}{r}} .
$$

Using Minkowski's inequality and the fact that $f$ is generalized $r$-convex function, we have

$$
\begin{aligned}
\frac{1}{b-a} \int_{a}^{b} f(x) \mathrm{d} x & =\int_{0}^{1} f((1-t) a+t b) \mathrm{d} t \\
& \leq \int_{0}^{1}\left\{(1-t)[f(a)]^{r}+t[f(a)+\eta(f(b), f(a))]^{r}\right\}^{\frac{1}{r}} \mathrm{~d} t \\
& \leq\left\{\left(\int_{0}^{1}(1-t)^{\frac{1}{r}}[f(a)] \mathrm{d} t\right)^{r}+\left(\int_{0}^{1} t^{\frac{1}{r}}[f(a)+\eta(f(b), f(a))] \mathrm{d} t\right)^{r}\right\}^{\frac{1}{r}} \\
& =\left\{\left(\frac{r}{r+1}\right)^{r}\left([f(a)]^{r}+[f(a)+\eta(f(b), f(a))]^{r}\right)\right\}^{\frac{1}{r}} \\
& =\left(\frac{r}{r+1}\right)\left\{\left([f(a)]^{r}+[f(a)+\eta(f(b), f(a))]^{r}\right)\right\}^{\frac{1}{r}}
\end{aligned}
$$

which is the required result. 
Corollary 3.1. [29] If $\eta(f(b), f(a))=f(b)-f(a)$, then, under the assumptions of Theorem 3.7, we have

$$
\frac{1}{b-a} \int_{a}^{b} f(x) \mathrm{d} x \leq\left(\frac{r}{r+1}\right)\left\{\left(\left[f^{r}(a)+f^{r}(b)\right]\right)\right\}^{\frac{1}{r}} .
$$

Theorem 3.2. Let $f: I \rightarrow \mathbb{R}$ be generalized $r$-convex function on $I$. Then for $0<r \leq 1$, we have

$$
\begin{aligned}
& f^{r}\left(\frac{a+b}{2}\right)-\frac{1}{2(b-a)} \int_{a}^{b} \eta(f(a+b-x), f(x)]^{r} \mathrm{~d} x \leq \frac{1}{2(b-a)} \int_{a}^{b} f^{r}(x) \mathrm{d} x \\
\leq & \left\{\frac{\left\{[f(a)]^{r}+[f(b)]^{r}\right\}}{4}+\frac{1}{4}\left([f(a)+\eta(f(b), f(a))]^{r}+[f(b)+\eta(f(a), f(b))]^{r}\right)\right\} .
\end{aligned}
$$

Proof. Let $f$ be generalized $r$-convex function on $I$. Then for $t=\frac{1}{2}$, we have

$$
f^{r}\left(\frac{x+y}{2}\right) \leq\left\{\frac{[f(x)]^{r}+[f(x)+\eta(f(y), f(x))]^{r}}{2}\right\} .
$$

This implies that

$$
\begin{aligned}
& f^{r}\left(\frac{a+b}{2}\right) \\
& \leq\left\{\frac{[f((1-t) a+t b)]^{r}+[f((1-t) a+t b)+\eta(f(t a+(1-t) b), f((1-t) a+t b))]^{r}}{2}\right\} .
\end{aligned}
$$

Integrating the above inequality with respect to $t$ on $[0,1]$, we have

$$
\begin{aligned}
f^{r}\left(\frac{a+b}{2}\right) \leq & \frac{1}{2} \int_{0}^{1}\left\{[f((1-t) a+t b)]^{r}+[f((1-t) a+t b)\right. \\
& \left.+\eta(f(t a+(1-t) b), f((1-t) a+t b))]^{r}\right\} \mathrm{d} t \\
= & \frac{1}{2(b-a)} \int_{a}^{b}\left\{[f(x)]^{r}+[f(x)+\eta(f(a+b-x), f(x))]^{r}\right\} \mathrm{d} x .
\end{aligned}
$$

This implies

$$
\begin{aligned}
& f^{r}\left(\frac{a+b}{2}\right)-\frac{1}{2(b-a)} \int_{a}^{b}[f(x)+\eta(f(a+b-x), f(x))]^{r} \mathrm{~d} x \\
& \leq \frac{1}{2(b-a)} \int_{a}^{b} f^{r}(x) \mathrm{d} x .
\end{aligned}
$$

Consider,

$$
\begin{aligned}
& f^{r}((1-t) a+t b) \leq\left\{(1-t)[f(a)]^{r}+t[f(a)+\eta(f(b), f(a))]^{r}\right\}, \\
& f^{r}((1-t) b+t a) \leq\left\{(1-t)[f(b)]^{r}+t[f(b)+\eta(f(a), f(b))]^{r}\right\} .
\end{aligned}
$$

Adding (3.2) and (3.3), we have

$$
\begin{aligned}
f^{r}((1-t) a+t b)+f^{r}((1-t) b+t a) \leq & \left\{(1-t)[f(a)]^{r}+t[f(a)+\eta(f(b), f(a))]^{r}\right\} \\
& +\left\{(1-t)[f(b)]^{r}+t[f(b)+\eta(f(a), f(b))]^{r}\right\} .
\end{aligned}
$$


Integrating the above inequality with respect to $t$ on $[0,1]$, we have

$$
\begin{aligned}
& \frac{2}{b-a} \int_{a}^{b} f^{r}(x) \mathrm{d} x \\
& \leq \int_{0}^{1}\left\{(1-t)\left([f(a)]^{r}+[f(b)]^{r}\right)+t\left([f(a)+\eta(f(b), f(a))]^{r}\right.\right. \\
& \left.\left.+[f(b)+\eta(f(a), f(b))]^{r}\right)\right\} \mathrm{d} t,
\end{aligned}
$$

which implies that

$$
\begin{aligned}
& \frac{1}{b-a} \int_{a}^{b} f^{r}(x) \mathrm{d} x \\
\leq & \left\{\frac{\left\{[f(a)]^{r}+[f(b)]^{r}\right\}}{4}+\frac{1}{4}\left([f(a)+\eta(f(b), f(a))]^{r}\right.\right. \\
& \left.\left.+[f(b)+\eta(f(a), f(b))]^{r}\right)\right\} .
\end{aligned}
$$

Combining (3.1) and (3.4), we have

$$
\begin{aligned}
& f^{r}\left(\frac{a+b}{2}\right)-\frac{1}{2(b-a)} \int_{a}^{b}[f(x)+\eta(f(a+b-x), f(x))]^{r} \mathrm{~d} x \leq \frac{1}{2(b-a)} \int_{a}^{b} f^{r}(x) \mathrm{d} x \\
\leq & \left\{\frac{\left\{[f(a)]^{r}+[f(b)]^{r}\right\}}{4}+\frac{1}{4}\left([f(a)+\eta(f(b), f(a))]^{r}+[f(b)+\eta(f(a), f(b))]^{r}\right)\right\},
\end{aligned}
$$

which is the required result.

Corollary 3.2. [29] If $\eta(f(b), f(a))=f(b)-f(a)$, then, under the assumptions of Theorem 3.2, we have

$$
f^{r}\left(\frac{a+b}{2}\right) \leq \frac{1}{b-a} \int_{a}^{b} f^{r}(x) \mathrm{d} x \leq\left\{\frac{\left[f^{r}(a)+f^{r}(b)\right]}{2}\right\} .
$$

Theorem 3.3. Let $f: I \rightarrow \mathbb{R}$ be generalized $r$-convex function on $I$ and $r \geq 0$, then

$$
\frac{1}{b-a} \int_{a}^{b} f(x) \mathrm{d} x \leq \begin{cases}\left(\frac{r}{r+1}\right)\left\{\frac{\eta\left(f^{r+1}(b), f^{r+1}(a)\right)}{\eta\left(f^{r}(b), f^{r}(a)\right)}\right\}, & r \neq 0 \\ \left\{\frac{[f(a)+\eta(f(b), f(a))]-[f(a)]}{\log [f(a)+\eta(f(b), f(a))]-\log [f(a)]}\right\}, & r=0 .\end{cases}
$$

Proof. First, let $r>0$ and $f$ be generalized $r$-convex function on $I$. Then, $\forall a, b \in I, t \in[0,1]$, we have

$$
\begin{aligned}
\frac{1}{b-a} \int_{a}^{b} f(x) \mathrm{d} x & =\int_{0}^{1} f((1-t) a+t b) \mathrm{d} t \\
& \leq \int_{0}^{1}\left\{(1-t)[f(a)]^{r}+t[f(a)+\eta(f(b), f(a))]^{r}\right\}^{\frac{1}{r}} \mathrm{~d} t
\end{aligned}
$$

Subsituting $u=\left[(1-t)[f(a)]^{r}+t[f(a)+\eta(f(b), f(a))]^{r}\right]$ in $(3.5)$, we have

$$
\begin{aligned}
\frac{1}{b-a} \int_{a}^{b} f(x) \mathrm{d} x & \leq \frac{1}{[f(a)+\eta(f(b), f(a))]^{r}-[f(a)]^{r}} \int_{[f(a)]^{r}}^{[f(a)+\eta(f(b), f(a))]^{r}} u^{\frac{1}{r}} \mathrm{~d} u \\
& =\left(\frac{r}{r+1}\right)\left\{\frac{\left([f(a)+\eta(f(b), f(a))]^{r+1}-[f(a)]^{r+1}\right)}{[f(a)+\eta(f(b), f(a))]^{r}-[f(a)]^{r}}\right\}
\end{aligned}
$$


For $r=0$, we have

$$
f((1-t) a+t b) \leq\left\{[f(a)]^{1-t}[f(a)+\eta(f(b), f(a))]^{t}\right\} .
$$

Hence we have,

$$
\begin{aligned}
\frac{1}{b-a} \int_{a}^{b} f(x) \mathrm{d} x & =\int_{0}^{1} f((1-t) a+t b) \mathrm{d} t \\
& \leq \int_{0}^{1}\left\{[f(a)]^{1-t}[f(a)+\eta(f(b), f(a))]^{t}\right\} \mathrm{d} t \\
& =[f(a)] \int_{0}^{1}\left\{\frac{[f(a)+\eta(f(b), f(a))]}{[f(a)]}\right\}^{t} \mathrm{~d} t \\
& =\left\{\frac{[f(a)+\eta(f(b), f(a))]-[f(a)]}{\log [f(a)+\eta(f(b), f(a))]-\log [f(a)]}\right\},
\end{aligned}
$$

which is the required result.

Corollary 3.3. [8] If $\eta(f(b), f(a))=f(b)-f(a)$, then, under the assumptions of Theorem 3.3, we have

$$
\frac{1}{b-a} \int_{a}^{b} f(x) \mathrm{d} x \leq \begin{cases}\left(\frac{r}{r+1}\right)\left\{\frac{\left(f^{r+1}(b)-f^{r+1}(a)\right)}{\left(f^{r}(b)-f^{r}(a)\right)}\right\}=L_{r}(f(a), f(b)), & r \neq 0 \\ \left\{\frac{[f(b)-f(a)]}{\log [f(b)]-\log [f(a)]}\right\}=L(f(a), f(b)), & r=0 .\end{cases}
$$

Theorem 3.4. Let $f: I \rightarrow \mathbb{R}$ be generalized $r$-convex function on $I$ and $r \geq 0$, then

$$
\frac{1}{b-a} \int_{a}^{b} f(x) \mathrm{d} x \leq\left\{\begin{array}{l}
\left.\left.\left\{\left[f^{r}(a)\right]+\left(\frac{r}{r+1}\right)^{r} \eta\left(f^{r}(a), f^{r}(a)\right)\right]\right)\right\}^{\frac{1}{r}}, r \neq 0, f(a)=f(b), \\
\frac{1}{\eta\left(f^{-1}(b), f^{-1}(a)\right)}\left[\log \left[f^{-1}(a)+\eta\left(f^{-1}(b), f^{-1}(a)\right)\right]-\log \left[f^{-1}(a)\right]\right], \quad r=-1 .
\end{array}\right.
$$

Proof. First, let $r>0, f$ be generalized $r$-convex function on $I$ and $f(a)=f(b)$. Then, $\forall a, b \in I, t \in[0,1]$, we have

$$
\begin{aligned}
\frac{1}{b-a} \int_{a}^{b} f(x) \mathrm{d} x & =\int_{0}^{1} f((1-t) a+t b) \mathrm{d} t \\
& \leq \int_{0}^{1}\left\{(1-t)[f(a)]^{r}+t[f(a)+\eta(f(a), f(a))]^{r}\right\}^{\frac{1}{r}} \mathrm{~d} t \\
& =f(a)
\end{aligned}
$$

For $r=-1$ and $f(a) \neq f(b)$, we have 


$$
\begin{aligned}
\frac{1}{b-a} \int_{a}^{b} f(x) \mathrm{d} x & =\int_{0}^{1} f((1-t) a+t b) \mathrm{d} t \\
& \leq \int_{0}^{1}\left\{(1-t)[f(a)]^{-1}+t[f(a)+\eta(f(b), f(a))]^{-1}\right\}^{-1} \mathrm{~d} t \\
& =\frac{1}{[f(a)+\eta(f(b), f(a))]^{-1}-[f(a)]^{-1}} \int_{[f(a)]^{-1}}^{[f(a)+\eta(f(b), f(a))]^{-1}} \frac{1}{u} \mathrm{~d} u \\
& =\frac{\left[\log [f(a)+\eta(f(b), f(a))]^{-1}-\log [f(a)]^{-1}\right]}{[f(a)+\eta(f(b), f(a))]^{-1}-[f(a)]^{-1}}
\end{aligned}
$$

which is the required result.

Corollary 3.4. [29] If $\eta(f(b), f(a))=f(b)-f(a)$, then, under the assumptions of Theorem 3.4, we have

$$
\frac{1}{b-a} \int_{a}^{b} f(x) \mathrm{d} x \leq\left\{\begin{array}{l}
f(a), r \neq 0, f(a)=f(b) \\
f(a) f(b) \frac{\log [f(b)]-\log [f(a)]}{f(b)-f(a)}=L_{-1}(f(a), f(b), \quad r=-1
\end{array}\right.
$$

Theorem 3.5. Let $f, g: I \rightarrow \mathbb{R}$ be generalized $r_{1}$-convex function and generalized $r_{2}$-convex function respectively on $I$. Then for $r_{1}>0, r_{2}>0>$, we have

$$
\begin{aligned}
\frac{1}{b-a} \int_{a}^{b} f(x) g(x) \mathrm{d} x \leq & \left(\frac{r}{r+1}\right)\left\{\left([f(a)]^{r_{1}}+[f(a)+\eta(f(b), f(a))]^{r_{1}}\right)\right\}^{\frac{2}{r_{1}}} \\
& +\left(\frac{r}{r+1}\right)\left\{\left([g(a)]^{r_{2}}[g(a)+\eta(g(b), g(a))]^{r_{2}}\right)\right\}^{\frac{2}{r_{2}}}
\end{aligned}
$$

Proof. Let $f, g: I \rightarrow \mathbb{R}$ be generalized $r_{1}$-convex function and generalized $r_{2}$-convex function respectively on $I$ with $\left(r_{1}>0, r_{2}>0\right)$. Then, $\forall a, b \in I, t \in[0,1]$, we have

$$
\begin{aligned}
& f((1-t) a+t b) \leq\left\{(1-t)[f(a)]^{r_{1}}+t[f(a)+\eta(f(b), f(a))]^{r_{1}}\right\}^{\frac{1}{r_{1}}} \\
& g((1-t) a+t b) \leq\left\{(1-t)[g(a)]^{r_{2}}+t[g(a)+\eta(g(b), g(a))]^{r_{2}}\right\}^{\frac{1}{r_{2}}}
\end{aligned}
$$


Using Cauchy's and Minkowski's inequalities and the fact that $f$ and $g$ are generalized $r_{1}$ and $r_{2}$-convex functions, we have

$$
\begin{aligned}
& \frac{1}{b-a} \int_{a}^{b} f(x) g(x) \mathrm{d} x=\int_{0}^{1} f((1-t) a+t b) g((1-t) a+t b) \mathrm{d} t \\
& \left.\leq \int_{0}^{1}\left\{(1-t)[f(a)]^{r_{1}}+t\left[f(a)+\eta\left(f^{(} b\right), f(a)\right)\right]^{r_{1}}\right\}^{\frac{1}{r_{1}}} \\
& \left\{(1-t)[g(a)]^{r_{2}}+t[g(a)+\eta(g(b), g(a))]^{r_{2}}\right\}^{\frac{1}{r_{2}}} \mathrm{~d} t \\
& \leq \frac{1}{2} \int_{0}^{1}\left\{(1-t)[f(a)]^{r_{1}}+t[f(a)+\eta(f(b), f(a))]^{r_{1}}\right\}^{\frac{2}{r_{1}}} \mathrm{~d} t \\
& +\frac{1}{2} \int_{0}^{1}\left\{(1-t)[g(a)]^{r_{2}}+t[g(a)+\eta(g(b), g(a))]^{r_{2}}\right\}^{\frac{2}{r_{2}}} \mathrm{~d} t \\
& \leq \frac{1}{2}\left\{\left(\int_{0}^{1}(1-t)^{\frac{2}{r_{1}}}[f(a)]^{2} \mathrm{~d} t\right)^{\frac{r_{1}}{2}}\right. \\
& \left.+\left(\int_{0}^{1} t^{\frac{2}{r_{1}}}[f(a)+\eta(f(b), f(a))]^{2} \mathrm{~d} t\right)^{\frac{r_{1}}{2}}\right\}^{\frac{2}{r_{1}}} \\
& +\frac{1}{2}\left\{\left(\int_{0}^{1}(1-t)^{\frac{2}{r_{2}}}[g(a)]^{2} \mathrm{~d} t\right)^{\frac{r_{2}}{2}}\right. \\
& \left.+\left(\int_{0}^{1} t^{\frac{2}{r_{2}}}[g(a)+\eta(g(b), g(a))]^{2} \mathrm{~d} t\right)^{\frac{r_{2}}{2}}\right\}^{\frac{2}{r_{2}}} \\
& =\left\{\left(\frac{r}{r+1}\right)^{\frac{r_{1}}{2}}\left([f(a)]^{r_{1}}+[f(a)+\eta(f(b), f(a))]^{r_{1}}\right)\right\}^{\frac{2}{r_{1}}} \\
& +\left\{\left(\frac{r}{r+1}\right)^{\frac{r_{2}}{2}}\left([g(a)]^{r_{2}}[g(a)+\eta(g(b), g(a))]^{r_{2}}\right)\right\}^{\frac{2}{r_{2}}} \\
& =\left(\frac{r}{r+1}\right)\left\{\left([f(a)]^{r_{1}}+[f(a)+\eta(f(b), f(a))]^{r_{1}}\right)\right\}^{\frac{2}{r_{1}}} \\
& +\left(\frac{r}{r+1}\right)\left\{\left([g(a)]^{r_{2}}[g(a)+\eta(g(b), g(a))]^{r_{2}}\right)\right\}^{\frac{2}{r_{2}}},
\end{aligned}
$$

which is the required result.

Corollary 3.5. [29] If $\eta(f(b), f(a))=f(b)-f(a)$, then, under the assumptions of Theorem 3.5, we have

$$
\begin{aligned}
\frac{1}{b-a} \int_{a}^{b} f(x) g(x) \mathrm{d} x \leq & \left(\frac{r}{r+1}\right)\left\{\left(\left[f^{r_{1}}(a)+f^{r_{1}}(b)\right]\right)\right\}^{\frac{2}{r_{1}}} \\
& +\left(\frac{r}{r+1}\right)\left\{\left(\left[g^{r_{2}}(a)+g^{r_{2}}(b)\right]\right)\right\}^{\frac{2}{r_{2}}} .
\end{aligned}
$$


Theorem 3.6. Let $f, g: I \rightarrow \mathbb{R}$ be generalized $r_{1}$-convex function and generalized $r_{2}$-convex function respectively on I. Then for $r_{1}>0, r_{2}>0$ and $\frac{1}{r_{1}}+\frac{1}{r_{2}}=1$, we have

$$
\begin{aligned}
\frac{1}{b-a} \int_{a}^{b} f(x) \mathrm{d} x \leq & \frac{1}{2}\left\{\left([f(a)]^{r_{1}}+[f(a)+\eta(f(b), f(a))]^{r_{1}}\right)^{\frac{1}{r_{1}}}\right. \\
& \left.\left([g(a)]^{r_{2}}+\left[g^{r_{2}}(a)+\eta(g(b), g(a))\right]^{r_{2}}\right)^{\frac{1}{r_{1}}}\right\} .
\end{aligned}
$$

Proof. Let $f, g: I \rightarrow \mathbb{R}$ be generalized $r_{1}$-convex function and generalized $r_{2}$-convex function respectively on $I$ with $\left(r_{1}>0, r_{2}>0\right)$. Then, $\forall a, b \in I, t \in[0,1]$, we have

$$
\begin{aligned}
& f((1-t) a+t b) \leq\left\{(1-t)[f(a)]^{r_{1}}+t[f(a)+\eta(f(b), f(a))]^{r_{1}}\right\}^{\frac{1}{r_{1}}} \\
& g((1-t) a+t b) \leq\left\{(1-t)[g(a)]^{r_{2}}+t[g(a)+\eta(g(b), g(a))]^{r_{2}}\right\}^{\frac{1}{r_{2}}} .
\end{aligned}
$$

Using Holder's inequality and the fact that $f$ and $g$ are generalized $r_{1}$ and $r_{2}$-convex functions, we have

$$
\begin{aligned}
\frac{1}{b-a} \int_{a}^{b} f(x) g(x) \mathrm{d} x= & \int_{0}^{1} f((1-t) a+t b) g((1-t) a+t b) \mathrm{d} t \\
\leq & \int_{0}^{1}\left\{(1-t)[f(a)]^{r_{1}}+t[f(a)+\eta(f(b), f(a))]^{r_{1}}\right\}^{\frac{1}{r_{1}}} \\
& \left\{(1-t)[g(a)]^{r_{2}}+t[g(a)+\eta(g(b), g(a))]^{r_{2}}\right\}^{\frac{1}{r_{2}}} \mathrm{~d} t \\
\leq & \left\{\int_{0}^{1}(1-t)[f(a)]^{r_{1}}+t[f(a)+\eta(f(b), f(a))]^{r_{1}} \mathrm{~d} t\right\}^{\frac{1}{r_{1}}} \\
& \left\{\int_{0}^{1}(1-t)[g(a)]^{r_{2}}+t[g(a)+\eta(g(b), g(a))]^{r_{2}} \mathrm{~d} t\right\}^{\frac{1}{r_{2}}} \\
= & \left\{\left([f(a)]^{r_{1}} \int_{0}^{1}(1-t) \mathrm{d} t+[f(a)+\eta(f(b), f(a))]^{r_{1}} \int_{0}^{1} t \mathrm{~d} t\right)\right\}^{\frac{1}{r_{1}}} \\
& \left\{\left([g(a)]^{r_{2}} \int_{0}^{1}(1-t) \mathrm{d} t+[g(a)+\eta(g(b), g(a))]^{r_{2}} \int_{0}^{1} t \mathrm{~d} t\right)\right\}^{\frac{1}{r_{2}}} \\
= & \frac{1}{2}\left\{\left([f(a)]^{r_{1}}+[f(a)+\eta(f(b), f(a))]^{r_{1}}\right)^{\frac{1}{r_{1}}}\right. \\
& \left.\left([g(a)]^{r_{2}}+\left[g^{r_{2}}(a)+\eta(g(b), g(a))\right]^{r_{2}}\right)^{\frac{1}{r_{1}}}\right\},
\end{aligned}
$$

which is the required result.

Corollary 3.6. [29] If $\eta(f(b), f(a))=f(b)-f(a)$, then, under the assumptions of Theorem 3.6, we have

$$
\frac{1}{b-a} \int_{a}^{b} f(x) \mathrm{d} x \leq \frac{\left\{\left(\left[f^{r_{1}}(a)+f^{r_{1}}(b)\right]\right)^{\frac{1}{r_{1}}}\left(\left[g^{r_{2}}(a)+g^{r_{2}}(b)\right)^{\frac{1}{r_{1}}}\right\}\right.}{2} .
$$


Theorem 3.7. Let $f, g: I \rightarrow \mathbb{R}$ be generalized $r$-convex function on $I$. Then for $r>0$, we have

$$
\left(\frac{1}{b-a} \int_{a}^{b} f(x) g(x) \mathrm{d} x\right)^{r} \leq\left\{M(a, b)\left(\frac{r}{r+2}\right)^{r}+N(a, b)\left(\beta\left(\frac{1}{r}+1, \frac{1}{r}+1\right)\right)^{r}\right\}
$$

where

$$
\begin{aligned}
& M(a, b)=\left(\left[f^{r}(a) g^{r}(a)\right]+[f(a)+\eta(f(b), f(a))]^{r}[g(a)+\eta(g(b), g(a))]^{r}\right) \\
& N(a, b)=\left(\left[f^{r}(a)\right][g(a)+\eta(g(b), g(a))]^{r}+\left[g^{r}(a)\right][f(a)+\eta(f(b), f(a))]^{r}\right),
\end{aligned}
$$

and $\beta(\cdot, \cdot)$ is the Beta function.

Proof. Let $f, g$ be two generalized $r$-convex functions on $I$. Then $\forall a, b \in I, t \in[0,1]$, we have

$$
\begin{aligned}
& f((1-t) a+t b) \leq\left\{(1-t)[f(a)]^{r}+t[f(a)+\eta(f(b), f(a))]^{r}\right\}^{\frac{1}{r}} \\
& g((1-t) a+t b) \leq\left\{(1-t)[g(a)]^{r}+t[g(a)+\eta(g(b), g(a))]^{r}\right\}^{\frac{1}{r}} .
\end{aligned}
$$

Using Minkowski's inequality and the fact that $f$ and $g$ are generalized $r$-convex functions, we have

$$
\begin{aligned}
& \left(\frac{1}{b-a} \int_{a}^{b} f(x) g(x) \mathrm{d} x\right)^{r} \\
= & \left(\int_{0}^{1} f((1-t) a+t b) g((1-t) a+t b) \mathrm{d} t\right)^{r} \\
\leq & \left\{\int _ { 0 } ^ { 1 } \left((1-t)[f(a)]^{r}+t[f(a)+\eta(f(b), f(a))]^{r}\right.\right. \\
& \left.\left.(1-t)[g(a)]^{r}+t[g(a)+\eta(g(b), g(a))]^{r}\right)^{\frac{1}{r}} \mathrm{~d} t\right\}^{r} \\
= & \left\{\int _ { 0 } ^ { 1 } \left((1-t)^{2}\left[f^{r}(a) g^{r}(a)\right]+t(1-t)\right.\right. \\
& \left(\left[f^{r}(a)\right][g(a)+\eta(g(b), g(a))]^{r}+\left[g^{r}(a)\right][f(a)+\eta(f(b), f(a))]^{r}\right) \\
& \left.+t^{2}\left([f(a)+\eta(f(b), f(a))]^{r}[g(a)+\eta(g(b), g(a))]^{r}\right)\right\}^{r}
\end{aligned}
$$




$$
\begin{aligned}
\leq & \left\{\left[f^{r}(a) g^{r}(a)\right]\left(\int_{0}^{1}(1-t)^{\frac{2}{r}} \mathrm{~d} t\right)^{r}\right. \\
& +\left(\left[f^{r}(a)\right][g(a)+\eta(g(b), g(a))]^{r}+\left[g^{r}(a)\right][f(a)+\eta(f(b), f(a))]^{r}\right) \\
& \left(\int_{0}^{1}[t(1-t)]^{\frac{1}{r}} \mathrm{~d} t\right)^{r} \\
+ & \left([f(a)+\eta(f(b), f(a))]^{r}[g(a)+\eta(g(b), g(a))]^{r}\right)\left(\int_{0}^{1} t^{\frac{2}{r}} \mathrm{~d} t\right)^{r} \\
= & \left(\left[f^{r}(a) g^{r}(a)\right]+[f(a)+\eta(f(b), f(a))]^{r}[g(a)+\eta(g(b), g(a))]^{r}\right) \\
& \left(\int_{0}^{1} t^{\frac{2}{r}} \mathrm{~d} t\right)^{r} \\
& +\left(\left[f^{r}(a)\right]\left[g^{r}(a)+\eta\left(g^{r}(b), g^{r}(a)\right)\right]+\left[g^{r}(a)\right]\left[f^{r}(a)+\eta\left(f^{r}(b), f^{r}(a)\right)\right]\right) \\
& \left(\int_{0}^{1}[t(1-t)]^{\frac{1}{r}} \mathrm{~d} t\right)^{r} \\
= & \left\{M(a, b)\left(\frac{r}{r+2}\right)^{r}+N(a, b)\left(\beta\left(\frac{1}{r}+1, \frac{1}{r}+1\right)\right)^{r}\right\},
\end{aligned}
$$

which is the required result.

\section{ACKNOWLedGements}

The authors would like to thank the Rector, COMSATS Institute of Information Technology, Pakistan, for providing excellent research and academic environments.

\section{REFERENCES}

[1] G. D. Anderson, M. K. Vamanamurthy and M. Vuorinen, Generalized convexity and inequalities, J. Math. Anal. Appl, 335(2007), 1294-1308.

[2] M. Alomari, M. Darus and S. S. Dragomir, New inequalities of Simpson's type for s-convex functions with applications, RGMIA Res. Rep. Coll, 12 (4) (2009).

[3] G. Cristescu, L. Lupsa, Non-connected Convexities and Applications, Kluwer Academic Publishers, Dordrechet, Holland, (2002).

[4] M. R. Delavar and S. S. Dragomir, On $\eta$-convexity, Math. Inequal. Appl., 20(1)(2017), 203-216.

[5] S. S. Dragomir and C. E. M. Pearce, Selected topics on Hermite-Hadamard inequalities and applications, Victoria University, Australia, (2000).

[6] M. E. Gordji, M. R. Delavar and M. D. Sen, On $\varphi$ convex functions, J. Math. Inequal, 10(1)(2016), 173-183.

[7] M. E. Gordji, M. R. Delavar and S. S. Dragomir, An inequality related to $\eta$-convex functions (II), Int. J. Nonlinear. Anal. Appl, 6(2)(2015), 27-33.

[8] P. M. Gill, C. E. M. Pearce , J. Pecaric, Hadamards inequality for r-convex functions, J. Math. Anal. Appl, 215(1997), 461-470.

[9] J. Hadamard, Etude sur les proprietes des fonctions entieres e.t en particulier dune fonction consideree par Riemann, J.

Math. Pure. Appl., 58(1893), 171-215. 
[10] C. Hermite, Sur deux limites d'une integrale definie, Mathesis, 3(1883), 82.

[11] D. H. Hyers and S. M. Ulam, Approximately convex functions, Proc. Amer. Math. Soc, 3(1952), 821-828.

[12] C. P. Niculescu and L. E. Persson, Convex Functions and Their Applications. Springer-Verlag, New York, (2006).

[13] M. A. Noor, General variational inequalities, Appl. Math. Letters,1(1988), 119-121.

[14] M. A. Noor, Some develpments in general variational inequalities, Appl. Math. Comput. 152(2004), 199-277.

[15] M. A. Noor and K. I. Noor, Harmonic variational inequalities, Appl. Math. Inform. Sci. 10(5)(2016), 1811-1814.

[16] M. A. Noor and K. I. Noor, Some implicit methods for solving harmonic variational inequalities , Inter. J. Anal. Appl. 12(1)(2016), 10-14.

[17] M. A. Noor, K. I. Noor and M. U. Awan, Some new estimates of Hermite-Hadamard inequalities via harmonically $r$-convex functions, Le Mathematiche, LXXI(II)(2016), 117-127.

[18] M. A. Noor, K. I. Noor, M. U. Awan and F. Safdar, On strongly generalized convex functions, Filomat, 31(18)(2017), 5783-5790.

[19] M. A. Noor, K. I. Noor and F. Safdar, Generalized geometrically convex functions and inequalities, J. Inequal. Appl, 2017(2017), Article ID 22.

[20] M. A. Noor, K. I. Noor and F. Safdar, Integral inequaities via generalized convex functions, J. Math. Computer, Sci, $17(4)(2017), 465-476$.

[21] M. A. Noor, K. I. Noor, S. Iftikhar, F. Safdar, Integral inequaities for relative harmonic $(s, \eta)$-convex functions, Appl. Math. Comp. Sci, 1(1)(2015), 27-34.

[22] M. A. Noor, K. I. Noor and F. Safdar, Integral inequaities via generalized $(\alpha, m)$-convex functions, J. Nonlinear. Funct. Anal, 2017(2017), Article ID 32.

[23] M. A. Noor, K. I. Noor and F. Safdar, New inequalities for generalized log $h$-convexd functions, J. Appl. Math. Inform. 36(3-4)(2018), 245-256.

[24] M. A. Noor, K. I. Noor and F. Safdar,Inequalities via generalized beta $m$-convex functions, J. Math. Anal. 9((2018).

[25] M. A. Noor, K. I. Noor and S. Iftikhar, Inequaities via $(p, r)$-convex functions, RAD, (2018).

[26] M. A. Noor, K. I. Noor and S. Iftikhar, On harmonic $(h, r)$-convex functions, Proced. Jangj. Math. Soc. 21(2)(2018), 239-251.

[27] M. A. Noor, K. I. Noor, F. Safdar, M. U. Awan and S. Ullah, Inequaities via generalized log $m$-convex functions, J. Nonlinear. Sci. Appl, 10(2017), 5789-5802.

[28] M. A. Noor, K. I. Noor, S. Iftikhar and F. Safdar, Generalized $(h, r)$-harmonic convex functions and inequalities, Int. J. Math. Anal. 16(4)(2018).

[29] N. P. N. Ngoc, N.V. Vinh, P. T. T. Hien, Integral inequalities of Hadamard type for r-Convex functions, Int. Math. Forum, $4(35)(2009), 1723-1728$.

[30] J. Pecaric, F. Proschan and Y. T. Tong, Convex Functions, Partial Ordering and Statistical Applications, Academic Pres, New York, (1992).

[31] G. S. Yang, Refinement of Hadamard's inequality for $r$-convex functions, Indian J. Pure Appl. Math. 32(10)(2001), 15711579. 